\title{
The effect of semantic distance in yes/no and go/no-go semantic categorization tasks
}

\author{
PAUL D. SIAKALUK \\ University of Southern Mississippi, Hattiesburg, Mississippi \\ LORI BUCHANAN \\ University of Windsor, Windsor, Ontario, Canada \\ and \\ CHRIS WESTBURY \\ University of Alberta, Edmonton, Alberta, Canada
}

\begin{abstract}
The effect of semantic distance (Lund \& Burgess, 1996) was examined in three semantic categorization experiments. Experiment 1, a yes/no task that required participants to make animal/nonanimal judgments by responding to both sets of stimuli (Forster \& Shen, 1996), revealed no effect of semantic distance. Experiment 2, a go/no-go task that required participants to respond to only the experimental (i.e., nonanimal) items, revealed a large effect of semantic distance. In addition, response latencies were longer and error rates were lower to the experimental items in Experiment 2 than to those in Experiment 1. These findings were replicated in Experiment 3, in which semantic distance and task condition were manipulated within subjects. We conclude that these results are consistent with (1) the view that the go/no-go tasks elicited more extensive processing of the experimental items and (2) a connectionist account of semantic activation, whereby processing is facilitated by the presence of semantic neighbors.
\end{abstract}

How semantics affects the visual identification of printed words has long been of interest to psycholinguists (for a review, see Balota, Ferraro, \& Connor, 1991). One important facet of this research has been to identify different characteristics of semantics and to measure their influence on visual word recognition processes. Some examples of this research are studies investigating the influence of polysemy (e.g., Borowsky \& Masson, 1996; Hino \& Lupker, 1996), imagery (e.g., Cortese, Simpson, \& Woolsey, 1997; Strain, Patterson, \& Seidenberg, 1995), concreteness (e.g., James, 1975; Kroll \& Mervis, 1986), featural information (e.g., McRae, de Sa, \& Seidenberg, 1997; Pexman, Lupker, \& Hino, 2002), and synonymy (e.g., Hino, Lupker, \& Pexman, 2002; Pecher, 2001).

Recently, Burgess and colleagues (Burgess, Livesay, \& Lund, 1998; Burgess \& Lund, 2000; Lund \& Burgess, 1996) have added to the ways of conceptualizing semantics. They have developed the hyperspace analogue to lan-

This research was supported by a Natural Sciences and Engineering Research Council of Canada (NSERC) operating grant to L.B. and an NSERC Postgraduate Scholarship to P.D.S. The experiments were conducted as part of a doctoral dissertation by the first author. Portions of these data were presented at the 42nd Annual Meeting of the Psychonomic Society, Orlando, FL, November, 2001. We thank Michael Cortese, Steve Joordens, Manuel Perea, and Jay Rueckl for helpful comments on an earlier version of this manuscript. Correspondence concerning this article should be addressed to L. Buchanan, Department of Psychology, University of Windsor, Windsor, ON, N9B 3P4 Canada (email: buchanan@uwindsor.ca). guage (or HAL) model of semantic memory (see also Landauer \& Dumais, 1997, for a similar methodological and theoretical approach). HAL is a high-dimensional, computational model that uses a global co-occurrence learning algorithm to track lexical co-occurrences across a large sample of written text. More specifically, HAL uses a 20word moving window (10 words in either direction of the word under consideration) to track the co-occurrences of words. HAL generates weighted co-occurrence values for words that co-occur in the 20-word window, with adjacent words on either side receiving a value of 10 and words separated by nine others on either side receiving a value of 1 . HAL sums these co-occurrence values, and this summing results in a high-dimensional co-occurrence matrix containing information from the entire learning history of the model. Each word is thus represented by a vector containing many (i.e., 140,000 dimensions with 70,000 words) elements. Each element can be thought of as a coordinate in high-dimensional space: Words with similar vectors occur relatively close together in this high-dimensional space, whereas words with dissimilar vectors are more distant. A distance metric in units, called Riverside context units (RCUs; see Lund \& Burgess, 1996), expresses the distance between any two words in the high-dimensional space.

Burgess et al. (1998) have presented many simulations and empirical studies that have demonstrated how words are represented in the high-dimensional space. For example, they used multidimensional scaling to demonstrate that the words in the high-dimensional space are clustered 
Table 1

Examples of Semantic Neighbors for the High Semantic Distance Word Amaze and the Low Semantic Distance Word Accept

Amaze

$($ Mean Distance $=501.46 \mathrm{RCU})$

\begin{tabular}{lcllc}
\cline { 1 - 1 } \cline { 5 - 5 } Neighbor & RCU & & Neighbor & RCU \\
\hline avoid & 492.90 & & take & 218.08 \\
defend & 494.55 & & give & 223.49 \\
learn & 497.07 & & recognize & 231.75 \\
convince & 501.31 & & continue & 237.39 \\
keep & 501.53 & & follow & 241.95 \\
demonstrate & 502.41 & & make & 242.14 \\
prove & 502.47 & & keep & 247.30 \\
make & 507.00 & & acknowledge & 249.21 \\
remind & 507.24 & & speak & 249.45 \\
create & 508.19 & & use & 251.23 \\
\hline
\end{tabular}

together in coherent ways. They demonstrated that HAL vectors are able to categorize words representing different concrete concepts (e.g., foods, clothes, and vehicles), different abstract concepts (e.g., emotional words, such as love and romance, and legal words, such as judge and law), as well as grammatical concepts (verbs from nouns). Other work by Burgess et al. focuses on the actual constituents of a given word neighborhood. In the HAL model, a word's neighbors are the words that are closest to it in semantic space. If one is interested in finding, for example, the 10 closest neighbors to any word, the 10 words with the lowest RCU values are those 10 closest neighbors. An example of this is shown in Table 1 . The 10 closest neighbors of the words amaze and accept are listed from lowest to highest RCU value (i.e., from closest neighbor to farthest neighbor). Note that the two words differ in their average semantic distance to their 10 closest semantic neighbors. This will be discussed further in the next paragraph. Burgess et al. demonstrated that RCU values can predict participants' abilities to make discriminations between similar words and to generate words that may be the core concept of a particular neighborhood of words.

The primary focus of Burgess and his colleagues, thus far, has been to develop an account of a word's semantic representation. Integral to this account is that according to HAL, word meaning is a function of the contexts in which words appear (Burgess, 1998; Burgess et al., 1998). In addition, words in similar regions of the high-dimensional space are similar in meaning, and this may result for one of two reasons. First, words may co-occur together in sim- ilar contexts and, thus, have similar values on their vectors. Burgess et al. used the example of the words cop and arrested as not being similar because of item similarity (i.e., they do not share similar features, as the words lion and tiger do) but because they co-occur in similar contexts. Second, words may have similar vector values but rarely, if ever, co-occur. For example, the words road and street rarely co-occur, but they appear in similar contexts, and thus their locations in the high-dimensional space are similar. In summary, a word's position in HAL's highdimensional space is a function of summing all the contexts in which it appears, and words that appear (whether they co-occur or not) in similar contexts are located in similar regions of the high-dimensional space.

A recent study by Buchanan, Westbury, and Burgess (2001) extended the empirical study of semantic distance to determine its processing implications for the visual identification of single words. They defined the semantic distance of a word as the mean distance between that word and its 10 closest neighbors in semantic space. Thus, a word with a high semantic distance, such as amaze, is relatively distant from its 10 nearest neighbors and may, therefore, be said to have a sparse semantic neighborhood.Conversely, a word with a low semantic distance, such as accept, is relatively close to its 10 nearest neighbors and, hence, can be said to have a dense semantic neighborhood. Another way of thinking about semantic distance is to imagine that within a specified radius, high semantic distance words will, on average, have fewer semantic neighbors than will low semantic distance words. Thus, high semantic distance words will, on average, have smaller semantic neighborhoods than will low semantic distance words.

Table 2 shows summary information of correlations, for 969 words, between semantic distance and other, more familiar measures of semantics, to provide a bit more information regarding this relatively novel variable. The concreteness, imageability, and meaningfulness values were taken from the MRC Psycholinguistic Database (Coltheart, 1981). The number of associates values were taken from the Nelson, McEvoy, and Schreiber (1994) word association norms. The semantic distance values were taken from our own list of semantic distance values. It is clear that semantic distance does not capture the same information as object-based variables, such as imageability or concreteness. However, as Buchanan et al. (2001) suggested, semantic distance reflects linguistic relationships rather

Table 2

Single-Order Correlations of Concreteness, Imageability, Meaningfulness (Colorado Norms), Number of Word Associates, and Semantic Distance

\begin{tabular}{lccccr}
\hline & Conc & Imag & Mean & NWA & SemD \\
\hline Conc & 1.000 & & & & \\
Imag & $.895^{*}$ & 1.000 & & & \\
Mean & $.186^{*}$ & $.381^{*}$ & 1.000 & & \\
NWA & .001 & -.021 & .014 & 1.000 & \\
SemD & $.199^{*}$ & $.163^{*}$ & $-.184^{*}$ & $-.151^{*}$ & 1.000 \\
\hline
\end{tabular}

Note-Conc, concreteness; Imag, imageability; Mean, meaningfulness; NWA, number of word associates; SemD, semantic distance. ${ }^{*} p<.001$. 
than featural information and, as such, may be thought of as more similar to association norms than to these objectcentered semantic values.

A primary purpose of Buchanan et al.'s (2001) study was to examine whether semantic neighborhood size exerted an effect on the speed with which words were recognized. In addition to investigating the effect of semantic neighborhood size as measured by semantic distance, Buchanan et al. measured semantic neighborhood size, using the Nelson et al. (1994) word association norms. Thus, a second goal was to determine which, if either, of these two semantic neighborhood size measures better predicted word recognition performance.

In their first experiment, Buchanan et al. (2001) used regression techniques to address the issue of the effects of semantic neighborhood size in lexical decision. In their hierarchical regression analyses, they included log frequency, orthographic neighborhood size (Coltheart, Davelaar, Jonasson, \& Besner, 1977), letter length, semantic associate size, and semantic distance. They reported that semantic distance was the only semantic variable that predicted lexical decision latencies. Moreover, they reported a positive partial correlation, which reflected the fact that, as semantic distance decreased, so did response latency.

Buchanan et al. (2001) then included imageability ratings (taken from the MRC PsycholinguisticDatabase) for 584 of the original 750 words in their hierarchical regression analyses, to determine whether semantic distance accounted for response latency variance not accounted for by a more traditional semantic variable. They again reported that semantic distance predicted lexical decision latencies even when a traditional semantic variable (imageability) was considered in the analysis. Factorial experiments were used to further examine the effects of semantic distance, and the findings across experiments demonstrated large and robust effects of semantic distance in lexical decision, particularly for low-frequency words.

The results of Buchanan et al.'s (2001) study provide important preliminary evidence that suggests that semantic distance plays a role in the processing of single words in lexical decision. The lexical decision task, as well as another traditional word recognition task - the word naming task-have, however, been criticized because responses may be made without lexical selection taking place (e.g., Balota \& Chumbley, 1984, and Grainger \& Jacobs, 1996, for lexical decision; Coltheart, 1978, and Coltheart, Rastle, Perry, Langdon, \& Ziegler, 2001, for naming; see Andrews \& Heathcote, 2001, for a recent discussion of this topic). Thus, there are important reasons for finding a word recognition task that is sensitive to semantic influences and that results in data that are reflective of lexical selection.

Forster and Shen (1996) argued that the semantic categorization task demands lexical selection, because it requires that the meaning of a word be retrieved before correct classification can occur. In their version of the task, participants were required to determine whether presented words were the names of animals or not (see also Sears, Lupker, \& Hino, 1999). The participants were instructed to respond by pressing one key if the item was an animal name and another key if the item was not an animal name. We will refer to this task as the yes/no semantic categorization task, because participants were required to respond to both the animal name and the nonanimal name stimuli. Forster and Shen stated that one strength of this version of the semantic categorization task is that using a single category avoids having to present different category labels throughout the experiment (as in Balota \& Chumbley's, 1984, study; see also Balota \& Chumbley, 1990, Monsell, 1990, and Monsell, Doyle, \& Haggard, 1989, for further discussions of Balota \& Chumbley's, 1984, original semantic categorization task), and thus the complexity of the task is reduced (Bradley \& Forster, 1987). In addition, they stated that analyzing only responses to the nonanimal name items (i.e., the experimental words) minimizes any possible contaminating effects of semantic priming or category typicality.

\section{EXPERIMENT 1}

The purpose of the present experiment was to determine the effect of semantic distance in the yes/no semantic categorization task of Forster and Shen (1996). Because this task putatively requires unique word identification and, thus, the extraction of a word's meaning before it can be correctly classified, we thought it an ideal task to use to investigate whether semantic distance influences lexical selection processes.

\section{Method}

Participants. Thirty-six first-year undergraduate students from the University of Alberta participated in this experiment. All were native English speakers and reported that they had normal or correctedto-normal vision. All the participants received course credit.

Stimuli. The complete set of experimental words used in this experiment is presented in Appendix A, and the descriptive statistics for these stimuli are listed in Table 3 .

Forty monosyllabic low-frequency words with high semantic distance values and 40 monosyllabic low-frequency words with low semantic distance values were initially selected. The same definition of semantic distance as that used in Buchanan et al.'s (2001) study was used in the present study. That is, the semantic distance of a word was defined as the mean distance, as measured by RCUs, between that word and its 10 closest neighbors in semantic space (for examples, see Table 1). All of the words were either four letters or five letters in length, and all were of low printed frequency according to the printed word frequency norms of the CELEX database (Baayen, Piepenbrock, \& Gulikers, 1995).

Another 24 participants provided number-of-meaning ratings for these 80 words, following the procedure employed in Kellas, Ferraro, and Simpson (1988) and Hino and Lupker (1996). The 80 words, along with 40 nonwords, were randomly ordered, with 20 letter strings per page, and were presented in a questionnaire format (page order was randomized across participants). At the right hand of each letter string was a scale from 0 to 2 . The participants were asked to carefully read each letter string and to make a judgment as to how many meanings it possessed. The participants were asked to circle 0 when the letter string was judged to have no meaning, 1 when the letter string was judged to have one meaning, and 2 when the letter string was judged to have more than one meaning.

An additional two groups of 24 participants provided either concreteness ratings or imageability ratings for the 80 selected words. 
Table 3

Mean Semantic Distance, Printed Word Frequency, Number of Meanings, Concreteness Ratings, Imageability Ratings, Length in Letters, and Orthographic Neighborhood Size for the Word Stimuli Used in Experiments 1 and 2

\begin{tabular}{cccccccc}
\hline Condition & SemD & PWF & NoM & Conc & Imag & LL & N \\
\hline High SemD & 416.27 & 9.85 & 1.16 & 4.33 & 4.68 & 4.50 & 6.30 \\
Low SemD & 245.15 & 9.65 & 1.18 & 4.51 & 4.84 & 4.50 & 6.30 \\
\hline
\end{tabular}

Note-SemD, semantic distance; PWF, printed word frequency; NoM, number of meanings; Conc, concreteness; Imag, imageability; LL, length in letters; N, orthographic neighborhood size.

The instructions were identical to those used by Toglia and Battig (1978), except that different examples of words with high and low concreteness and imageability were presented. The 80 words were randomly ordered, with 20 words per page, and were presented in a questionnaire format (page order was randomized across participants). At the right of each letter string was a scale from 1 to 7 . The participants were asked to carefully read each word and rate it either as to its concreteness or its imageability.

From these ratings, 20 high semantic distance words and 20 low semantic distance words were chosen, equating as closely as possible the two word conditions for number of meanings, concreteness, and imageability. In addition to the above stimulus constraints, the items were matched as closely as possible for printed word frequency, letter length, and orthographic neighborhood size. As can be seen in Table 3, the high semantic distance words did not differ from the low semantic distance words on any of these variables $(p>.60$ in all cases).

An additional 40 words that were animal names were used in the experiment (these animal names are presented in Appendix B). Twenty of the animal names were four letters in length, and the remaining 20 animal names were five letters in length, thus being matched to the experimental items in letter length. A total of 80 words, therefore, were presented in the experiment. Following the instructions given by Forster and Shen (1996) and Sears, Lupker, and Hino (1999), the participants were explicitly told that the animal names included mammals, fish, reptiles, birds, amphibians, and insects but excluded humans or other types of living things (e.g., trees). They were further instructed that (1) the animal names could appear in the singular (e.g., spider), in the plural (e.g., lions), or in an informal or abbreviated version (e.g., bunny), (2) the animal names referred to the whole animal and not to a part of an animal (e.g., hoof), and (3) if the meaning of the word was ambiguous - that is, it could refer to an animal or not (e.g., tick) - they should consider these types of words as referring to the animal (although these types of animal names were avoided as much as possible)

Apparatus and Procedure. The stimuli were presented on a color VGA monitor driven by a Pentium-class microcomputer using the Micro Experimental Laboratory (MEL) software (Schneider, 1990). Response latencies were measured to the nearest millisecond.

For every trial, a 50-msec blank screen was followed by a fixation cross that appeared at the center of the computer monitor for 250 msec and was then replaced by a stimulus item (presented in lowercase letters). The participants responded animal by pressing the 1 key and nonanimal by pressing the 0 key on the computer keyboard. They were instructed to make their responses as quickly and as accurately as possible. The participant's response terminated the stimulus display, and the next trial was initiated after a timed interval of $1 \mathrm{sec}$ The order in which the experimental stimuli were presented in the experiment was randomized separately for each participant.

Each participant completed 16 practice trials prior to the collection of data. The practice stimuli consisted of eight words (half were four letters in length, half were five letters in length, and all were of the same frequency range as the experimental word stimuli), and eight animal names (half were four letters in length and half were five letters in length).
Design. A single-factor (semantic distance: high or low) design was used, with 20 words presented in each of the two semantic distance conditions. There were also 40 animal names presented, for a total of 80 trials. For the word data, response latencies and error rates from each participant were submitted to a single-factor (semantic distance: high or low) repeated measures analysis of variance (ANOVA). Both a within-subjects analysis $\left(F_{1}\right)$ and a between-items analysis $\left(F_{2}\right)$ were carried out.

\section{Results and Discussion}

Response latencies less than $250 \mathrm{msec}$ or more than $2,000 \mathrm{msec}$ were removed from the data set. A total of $0.1 \%$ of the data was removed by this procedure. For the experimental word and animal name stimuli, the mean response latencies of correct responses and the mean error rates are shown in Table 4. Unless noted, all effects are significant at $p<.05$.

In the analysis of the response latency data, the main effect of semantic distance was marginal in the subject analysis $\left[F_{1}(1,35)=3.93, M S_{\mathrm{e}}=1,087.21, p=.055\right]$, but not in the item analysis $\left[F_{2}(1,38)<1\right]$, with responses to the low semantic distance words an average of $15 \mathrm{msec}$ faster than responses to the high semantic distance words. In the analysis of the error rate data, there was no main effect of semantic distance $\left[F_{1}(1,35)<1 ; F_{2}(1,38)<1\right]$.

In the present experiment, which putatively elicits semantic processing so that the animal words and the nonanimal words can be correctly categorized, there was a hint of an effect of semantic distance on the response latency data in the subject analysis. This effect, however, was not found in the item analysis.

Semantic distance and subjective frequency. Conley, Burgess, and Decker's (2001) report of a correlation between subjective familiarity and semantic distance, combined with Balota and Chumbley's (1984) discussion regarding potential cross-task differences with respect to subjective familiarity effects, led us to consider this variable as a potential confound. To determine the subjective familiarity of the experimental items used in the present experiment, we used Balota, Pilotti, and Cortese's (2001) "subjective frequency" (their term, p. 640) ratings, which are based on a 7-point scale in which each point corresponds to a specific frequency of encounter for any given word. ${ }^{1}$ For example, 1 corresponds to never encountering the word, 4 corresponds to encountering the word once a week, and 7 corresponds to encountering the word several times a day. The experimental items were not matched on subjective frequency (3.32 and 3.75 for the high semantic 
Table 4

Mean Response Latencies (in Milliseconds) and Standard

Errors, and Mean Error Rates (Percentages) and Standard

Errors for the Word and Animal Stimuli in Experiments 1 and 2

\begin{tabular}{|c|c|c|c|c|c|}
\hline \multirow[b]{3}{*}{ Experiment } & \multicolumn{5}{|c|}{ Word Stimuli } \\
\hline & \multicolumn{2}{|c|}{$\begin{array}{l}\text { High Semantic } \\
\text { Distance }\end{array}$} & \multicolumn{2}{|c|}{$\begin{array}{l}\text { Low Semantic } \\
\text { Distance }\end{array}$} & \multirow{2}{*}{$\begin{array}{c}\text { Semantic Distance } \\
\text { Effect }\end{array}$} \\
\hline & $M$ & $\overline{S E}$ & $M$ & $S E$ & \\
\hline \multicolumn{6}{|c|}{ Response Latencies } \\
\hline 1: yes/no & 676 & 14.4 & 661 & 11.7 & 15 \\
\hline 2: go/no-go & 705 & 14.7 & 664 & 14.4 & 41 \\
\hline \multicolumn{6}{|c|}{ Error Rates } \\
\hline 1: yes/no & 2.6 & 0.8 & 3.1 & 0.8 & -0.5 \\
\hline 2: go/no-go & 0.5 & 0.3 & 0.4 & 0.2 & 0.1 \\
\hline \multicolumn{6}{|c|}{ Animal Names } \\
\hline 1: yes/no & 621 & 10.6 & 4.1 & 0.7 & \\
\hline 2: go/no-go & - & - & 5.6 & 1.0 & \\
\hline
\end{tabular}

distance words and the low semantic distance words, respectively). We regressed the semantic distance values of the experimental items against Balota et al.'s (2001) subjective frequency reading ratings and found that, consistent with Conley et al.'s report, the two variables were significantly correlated $(R=-.487)$; as semantic distance decreased, subjective frequency ratings increased.

We regressed the mean item response latencies against the subjective frequency ratings and found that subjective frequency accounted for only $1 \%$ of the response latency variance $(F<1)$. To determine whether semantic distance accounted for a significant amount of unique variance above and beyond that of subjective frequency, we subtracted the amount of variance accounted for by subjective frequency from the amount of variance accounted for by both subjective frequency and semantic distance (Green, 1978; Pedhazur, 1982). When both predictor variables were entered, they accounted for only $4 \%$ of the variance $(F<1)$. Semantic distance accounted for only $3 \%$ of the variance above and beyond subjective frequency $[F(1,37)=1.19$, $p>.10]$. This regression analysis coincides with our ANOVA analysis findings in that neither revealed an effect of semantic distance, but we can now be certain that this lack of effect was not due to confounds with subjective frequency.

We were initially surprised by this finding, given the robust nature of the semantic distance effect in lexical decision reported in Buchanan et al. (2001). Note, however, that if one compares the procedures of the lexical decision task and the yes/no semantic categorization task, there is one important difference. For lexical decision, the experimental words are the yes responses, whereas in semantic categorization these same items constitute the no responses (Carreiras, Perea, \& Grainger, 1997). In an attempt to bridge this methodological discrepancy, we conducted a go/nogo semantic categorization task in which the participants were required to respond only to the experimental items (i.e., the nonanimal name items). Our expectation was that this procedural change would make the responses to the experimental items more like yes responses, because they were the only items requiring an overt response, and that this would increase the likelihood of observing an effect of semantic distance.

\section{EXPERIMENT 2}

\section{Method}

Participants. Thirty-six first-year undergraduate students from the University of Alberta participated in the experiment for course credit. All were native English speakers and reported that they had normal or corrected-to-normal vision. None of these individuals had participated in the previous experiment or in the collection of any of the norming data of the previous experiment.

Stimuli. The same set of experimental word and animal name stimuli and the same instructions regarding the animal name stimuli as those used in Experiment 1 were used in this experiment.

Apparatus, Procedure, and Design. The apparatus, procedure, and design were the same as those in Experiment 1, with one change in procedure. The participants in this experiment were instructed to respond only if the stimulus was not an animal name. They responded to these items (i.e., the experimental words) by pressing the 0 key on the computer keyboard. The participants were instructed not to press any key on the computer keyboard if the stimulus was an animal name. They were told that the stimulus would remain on the monitor for $2.5 \mathrm{sec}$ and then it would be automatically replaced by the next stimulus item. Thus, they were instructed not to do anything if the stimulus item was an animal name, just to wait for it to be replaced by the next item. If they did respond by pressing the 0 key, the next item was presented after an interval of $1 \mathrm{sec}$. The order in which the experimental stimuli were presented in the experiment was randomized separately for each participant. Both a within-subjects analysis $\left(F_{1}\right)$ and a between-items analysis $\left(F_{2}\right)$ were carried out.

\section{Results and Discussion}

Response latencies less than $250 \mathrm{msec}$ or more than $2,000 \mathrm{msec}$ were removed from the data set. A total of $0.4 \%$ of the data was removed by this procedure. As for error rates, there was only one error made to the 40 experimental words across all 36 participants (in addition to the six outliers), and thus the analysis of error rates was not carried out. There were no mean response latency data 
to report for the animal stimuli, because the participants were instructed not to respond if the stimulus item was an animal name. The mean response latencies of correct responses, the mean error rates of the experimental words, and the mean error rates of the animal stimuli (false alarms, in this case) are shown in Table 4.

In the analysis of the response latency data, there was a main effect of semantic distance in both analyses $\left[F_{1}(1,35)=\right.$ $\left.48.55, M S_{\mathrm{e}}=607.72 ; F_{2}(1,38)=5.34, M S_{\mathrm{e}}=3,051.72\right]$, with responses to the low semantic distance words an average of $41 \mathrm{msec}$ faster than responses to the high semantic distance words.

To further investigate whether the effect of semantic distance was larger in the present experiment than in Experiment 1 , the response latency data from both experiments were submitted to a 2 (semantic distance: high or low) $\times 2$ (task condition: yes/no or go/no-go) mixedmodel ANOVA.

There was a main effect of semantic distance in the subject analysis $\left[F_{1}(1,70)=33.18, M S_{\mathrm{e}}=847.47\right]$, and this effect was marginal in the item analysis $\left[F_{2}(1,38)=3.11\right.$, $\left.M S_{\mathrm{e}}=4,785.80, p=.085\right]$. Responses to the low semantic distance words were an average of $28 \mathrm{msec}$ faster than responses to the high semantic distance words. There was a main effect of task condition in the item analysis $\left[F_{2}(1,38)=\right.$ $\left.5.70, M S_{\mathrm{e}}=779.59\right]$, but not in the subject analysis $\left[F_{1}(1,70)<1\right]$, with responses in Experiment 1 an average of $16 \mathrm{msec}$ faster than responses in Experiment 2. Most important, there was an interaction between semantic distance and task condition $\left[F_{1}(1,70)=6.68, M S_{\mathrm{e}}=\right.$ $\left.847.47 ; F_{2}(1,38)=4.39, M S_{\mathrm{e}}=779.59\right]$, since the size of the semantic distance effect almost tripled in the go/no-go task condition, as compared with the yes/no task condition (41 vs. $15 \mathrm{msec}$, respectively).

We conducted an analysis of the effects of subjective frequency identical to that described in Experiment 1. Subjective frequency accounted for only $4 \%$ of the response latency variance $[F(1,38)=1.60, p=.21]$. Semantic distance and subjective frequency, combined, accounted for $17 \%$ of the response latency variance $[F(2,37)=$ 3.86]. Finally, we subtracted the amount of variance accounted for by subjective frequency from the amount of variance accounted for by both subjective frequency and semantic distance (Green, 1978; Pedhazur, 1982) and found that semantic distance accounted for a unique $13 \%$ of the response latency variance $[F(1,37)=5.90]$. These regression analyses indicate that semantic distance exerted an increased effect in Experiment 2 and are entirely consistent with the findings based on ANOVAs. From these data, we can conclude that subjective frequency does not play as important a role in semantic categorization as does semantic distance. However, we note that it is quite possible that our stringent matching on word frequency reduced the range of subjective frequency to such an extent that a correlation with response latency would be difficult to obtain under any circumstances.

\section{EXPERIMENT 3}

The results of Experiments 1 and 2 demonstrated that semantic distance exerted a larger effect in the go/no-go version of the semantic categorization task than in the yes/no version of the task. We will postpone a detailed account of these results until the General Discussion section following Experiment 3, which addresses some methodological issues with respect to the cross-experiment analyses described above. A potential shortcoming of our design was that the participants were not randomly assigned to the different task conditions. This was because Experiment 2 was motivated by the lack of a robust effect of semantic distance in Experiment 1. To address this potential methodological problem, we carried out a third semantic categorization task, in which semantic distance and task procedure were manipulated within subjects.

\section{Method}

Participants. Thirty-six first-year undergraduate students from the University of Alberta participated in the experiment for course credit. All were native English speakers and reported that they had normal or corrected-to-normal vision. None of these individuals participated in the previous experiments or in the collection of norming data.

Stimuli. The complete set of experimental words used in this experiment is presented in Appendix $\mathrm{C}$, and the descriptive statistics for these stimuli are listed in Table 5 .

In order to include more items in each semantic distance condition, an additional 20 monosyllabic low-frequency words with high semantic distance values and 20 monosyllabic low-frequency words with low semantic distance values were selected. All of these 40 additional words were either four letters or five letters in length, and all were of low printed frequency according to the printed word frequency norms of the CELEX database (Baayen et al., 1995). Twenty-four participants provided number-of-meaning ratings for these 40 words, along with 20 nonwords, as was done previously (see the Method section for Experiment 1; the 60 items were randomly ordered, with 20 items per page, and were presented in a questionnaire format, with page order randomized across participants). Concreteness and imageability ratings for these words came from the MRC Psycholinguistic Database (Coltheart, 1981). ${ }^{2}$

Table 5

Mean Semantic Distance, Printed Word Frequency, Number of Meanings, Concreteness Ratings, Imageability Ratings, Length in Letters, Orthographic Neighborhood Size, and Subjective Frequency for the Word Stimuli Used in Experiment 3

\begin{tabular}{lcccccccr}
\hline Condition & SemD & PWF & NoM & Conc & Imag & LL & N & SubF \\
\hline High SemD & 402.69 & 9.00 & 1.22 & 456.00 & 486.06 & 4.40 & 6.83 & 3.69 \\
Low SemD & 243.94 & 9.13 & 1.31 & 472.73 & 493.26 & 4.40 & 6.76 & 3.87 \\
\hline
\end{tabular}

Note-SemD, semantic distance; PWF, printed word frequency; NoM, number of meanings; Conc, concreteness; Imag, imageability; LL, length in letters; N, orthographic neighborhood size; SubF, subjective frequency. 
As was the case in Experiments 1 and 2, the two experimental word conditions were equated as closely as possible for printed word frequency, number of meanings, concreteness, imageability, letter length, and orthographic neighborhood size. We also equated as closely as possible for subjective frequency, using Balota et al.'s (2001) subjective reading ratings. A total of 30 high semantic distance words and 30 low semantic distance words were selected for use in the present experiment (of these 60 items, 19 from each semantic distance condition were used in the previous experiments). As can be seen in Table 5, the high semantic distance words did not differ from the low semantic distance words on any of these variables ( $p>.20$ in all cases).

An additional 60 animal names were used in the experiment (these animal names are presented in Appendix B). Thirty-six of the animal names were four letters in length, and the remaining 24 were five letters in length; they thus matched the experimental items in letter length. A total of 120 items were therefore presented in the experiment. The same instructions regarding the animal stimuli as those used in Experiments 1 and 2 were used in this experiment.

Finally, Carreiras et al. (1997) reported small but significant correlations between experimental item response latencies and error rates and how "animal-like" their experimental items were, as measured with a 10-point animalness ratings scale. To investigate whether animalness influenced responses to the animal name and the nonanimal name items used in Experiment 3, we likewise collected animalness ratings, using a scale similar to that used by Carreiras et al. The 60 experimental words and the 60 animal names were randomly ordered, with 20 words per page, and were presented in a questionnaire format (page order was randomized across participants). To the right of each word was a scale from 1 to 10 , with 1 indicating not at all animal-like and 10 indicating very animal-like. Twenty participants were asked to rate how animal-like each word was. Not surprisingly, the animal name items had higher animalness ratings than did the experimental items ( 8.75 and 2.45 , respectively), and this difference was highly significant $[t(118)=33.69]$. Importantly, the high semantic distance words and the low semantic distance words were closely matched on this measure [ 2.38 and 2.51 , respectively; $t(58)=-0.51, p=.61]$.

Apparatus and Procedure. The stimuli were presented on an iMac computer using PsychLab software (Abrams, 1995). Response latencies were measured to the nearest millisecond.

Each participant saw half of the experimental items and half of the animal name items in a yes/no task condition and the other half of the experimental items and the other half of the animal name items in a go/no-go task condition. For every participant, the experimental items and the animal name items were randomly assigned to each task condition. Task condition was counterbalanced across participants, so that half of the participants had the yes/no task condition first and the go/no-go task condition second, whereas the other half of the participants had the go/no-go task condition first and the yes/no task condition second.

In the yes/no task condition, the participants responded animal by pressing the Command key and nonanimal by pressing the 0 key (from the number pad) on the computer keyboard. In the go/no-go task condition, the participants were instructed to respond only if the stimulus was not an animal name. They responded to these items (i.e., the experimental words) by pressing the 0 key (from the number pad) on the computer keyboard. The participants were instructed not to press any key on the computer keyboard if the stimulus was an animal name. They were told that the stimulus would remain on the monitor for $2.5 \mathrm{sec}$ and then it would be automatically replaced by the next stimulus item. Thus, they were instructed not to do anything if the stimulus item was an animal name, just to wait for it to be replaced by the next item. Stimulus items were presented in lowercase letters.

In the yes/no task condition, the participant's response terminated the stimulus display, and the next trial was initiated after a timed in- terval of $1 \mathrm{sec}$. In the go/no-go task condition, if the participant responded by pressing the $0 \mathrm{key}$, the next item was presented after an interval of $1 \mathrm{sec}$. If the participant did not respond, the stimulus item remained on the screen for $2.5 \mathrm{sec}$ and was then replaced by the next item. The participants were instructed to make their responses as quickly and as accurately as possible in both task conditions. The order in which the experimental stimuli were presented in the experiment was randomized separately for each participant.

To ensure that the participants were well practiced with each task condition, they completed 60 practice trials prior to the collection of data for each task condition. The practice stimuli consisted of 30 words (10 each consisting of four letters, five letters, and six letters, all from the same frequency range as the experimental word stimuli) and 30 animal names (10 each consisting of four letters, five letters, and six letters). There was a short break between task conditions.

Design. Semantic distance (high or low) and task condition (yes/no or go/no-go) were varied within subjects. The order of task condition was counterbalanced between participants, in that 18 participants completed the yes/no task condition first and the go/no-go task condition second, whereas the other 18 participants completed the go/no-go task condition first and the yes/no task condition second. Each participant responded to 60 stimuli in each task condition: 30 experimental items (15 high semantic distance words and 15 low semantic distance words) and 30 animal name items. For the word data, response latencies and error rates from each participant were submitted to a 2 (semantic distance: high or low) $\times 2$ (task condition: yes/no or go/no-go) repeated measures ANOVA. Both a within-subjects analysis $\left(F_{1}\right)$ and a between-items analysis $\left(F_{2}\right)$ were carried out.

\section{Results and Discussion}

Response latencies less than $250 \mathrm{msec}$ or more than $2,000 \mathrm{msec}$ were removed from the data set. A total of $0.3 \%$ of the yes/no responses and a total of $0.4 \%$ of the go/no-go responses were removed by this procedure. For the experimental word and animal name stimuli, the mean response latencies of correct responses and the mean error rates are shown in Table 6.

Response latencies. There was a main effect of semantic distance $\left[F_{1}(1,34)=14.59, M S_{\mathrm{e}}=2,346.41 ; F_{2}(1,58)=\right.$ $\left.6.92, M S_{\mathrm{e}}=88,828.66\right]$, with responses to the low semantic distance words an average of $31 \mathrm{msec}$ faster than responses to the high semantic distance words. There was a

\section{Table 6}

Mean Response Latencies (in Milliseconds) and Standard Errors and Mean Error Rates (Percentages) and Standard Errors for the Word and Animal Stimuli in Experiment 3

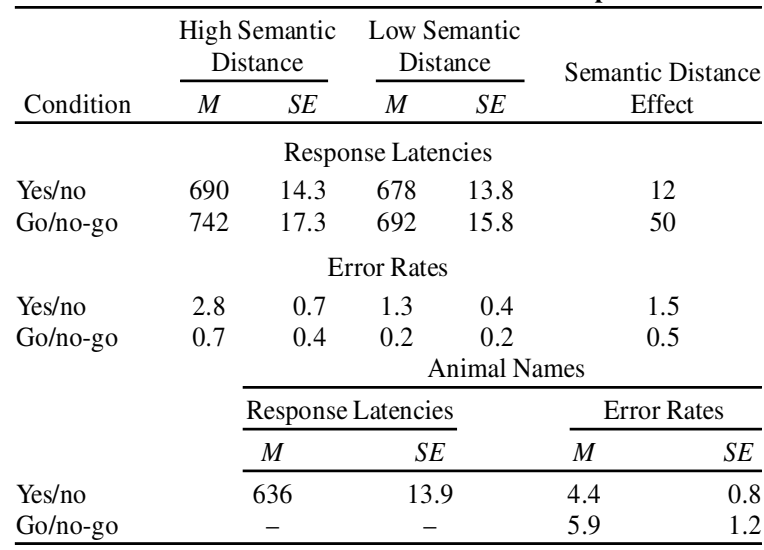


main effect of task condition $\left[F_{1}(1,34)=5.16, M S_{\mathrm{e}}=\right.$ $\left.7,564.52 ; F_{2}(1,58)=10.51, M S_{\mathrm{e}}=4,948.73\right]$, with responses in the go/no-go task condition an average of $33 \mathrm{msec}$ slower than responses in the yes/no task condition. There was also a main effect of order $\left[F_{1}(1,34)=4.92\right.$, $\left.M S_{\mathrm{e}}=19,433 \cdot 14 ; F_{2}(1,58)=4.76, M S_{\mathrm{e}}=5,622.46\right]$. Responses were $52 \mathrm{msec}$ slower when the go/no-go task condition was presented first than when the yes/no task condition was presented first.

There was a semantic distance $\times$ task condition interaction $\left[F_{1}(1,34)=5.69, M S_{\mathrm{e}}=2,230.84 ; F_{2}(1,58)=\right.$ $\left.4.40, M S_{\mathrm{e}}=4,948.73\right]$. There was no effect of semantic distance in the yes/no task condition $\left[t_{1}(34)=1.07, p>\right.$ $\left..10 ; t_{2}(58)=1.09, p>.10\right]$, but there was in the go/no-go task condition $\left[t_{1}(34)=4.49 ; t_{2}(58)=3.72\right]$. This interaction indicates that semantic distance exerted larger facilitatory effects in the go/no-go task condition $(50 \mathrm{msec})$ than in the yes/no task condition $(12 \mathrm{msec})$.

There was no task condition $\times$ order interaction in the subject analysis $\left[F_{1}(1,34)=2.86, M S_{\mathrm{e}}=7,564.57, p=\right.$ $.10]$, but there was in the item analysis $\left[F_{2}(1,58)=33.13\right.$, $\left.M S_{\mathrm{e}}=4,278.34\right]$. Post hoc comparisons of the item means revealed that in the yes/no task condition, responses were an average of $76 \mathrm{msec}$ faster when the yes/no task condition was presented first than when the go/no-go task condition was presented first $\left[t_{2}(58)=6.28\right]$. In the go/no-go task condition, responses were an average of $27 \mathrm{msec}$ faster when the yes/no task condition was presented first than when the go/no-go task condition was presented first $\left[t_{2}(58)=2.40\right]$. Importantly, there was no semantic distance $X$ order interaction, nor was there a three-way interaction $\left(F_{\mathrm{S}}<1\right.$ in all cases $)$.

The above analyses were conducted on mean reaction times. An objection to this approach may be that these values do not accurately reflect what may simply be an extension in the long tail of the response latency distribution in the case of the slower words. To address this potential objection, we contrasted the effects of semantic distance in the two task conditions, using median response latencies. These results mirrored the results of the mean response latency data. In fact, the magnitude of the semantic distance effect in the yes/no and the go/no-go task conditions when median response latencies were the dependent measure (18 and $51 \mathrm{msec}$, respectively) were similar to those when mean response latencies were the dependent measure (12 and $50 \mathrm{msec}$, respectively).

Error rates. There was a main effect of semantic distance in the subject analysis $\left[F_{1}(1,34)=6.14, M S_{\mathrm{e}}=6.55\right]$, but not in the item analysis $\left[F_{2}(1,58)=1.08, M S_{\mathrm{e}}=48.62\right.$, $p=.30]$. There were generally fewer errors made to the low semantic distance words than to the high semantic distance words ( $0.8 \%$ vs. $1.8 \%$, respectively). There was a main effect of task condition $\left[F_{1}(1,34)=12.93, M S_{\mathrm{e}}=\right.$ $\left.6.55 ; F_{2}(1,58)=8.53, M S_{\mathrm{e}}=21.59\right]$. More errors were made in the yes/no task condition than in the go/no-go task condition $(2.1 \%$ vs. $0.5 \%)$. There was no main effect of order (both $F_{\mathrm{S}}<1$ ).

There was no semantic distance $\times$ task condition interaction, nor was there a task condition $\times$ order interaction
( $F \mathrm{~s}<1$ in all cases). There was a marginal semantic distance $\times$ order interaction in the subject analysis $\left[F_{1}(1,34)=\right.$ $\left.3.40, M S_{\mathrm{e}}=6.55, p=.07\right]$, but not in the item analysis $\left[F_{2}(1,58)=2.73, M S_{\mathrm{e}}=21.59, p=.10\right]$. Finally, there was no three-way interaction in the subject analysis $\left(F_{1}<1\right)$, but it was marginal in the item analysis $\left[F_{2}(1,58)=2.73\right.$, $\left.M S_{\mathrm{e}}=18.93, p=.06\right]$.

Animalness ratings. We correlated the animalness ratings with the item response latency and error rate data of the animal name items and the experimental items separately. For the yes/no task condition data, there were significant correlations between the animal-likeness ratings and the animal name item response latencies and item error rates $(R=-.334$ and $R=-.438$, respectively). For the go/no-go task condition data, there was a significant correlation between the animal-likeness ratings and the animal name item error rates $(R=-.441)$. Note that the signs of the correlations were negative, indicating that as animalness ratings increased, response latencies and error rates to the animal name items decreased. Also note that the correlations between the animal-likeness ratings and the animal name error rates were virtually identical across the two task conditions. These findings are consistent with an explanation centered on early decisions regarding animallikeness enabling more rapid and more accurate responses to the animal name items with higher animal-likeness ratings than to the animal name items with lower animallikeness ratings. In contrast, the correlations between the animal-likeness ratings and the experimental item response latencies and item error rates were extremely small and nonsignificant. For the yes/no task condition, the correlations were $R=-.015$ and .137 , respectively, and for the go/no-go task condition, the correlations were $R=$ .172 and .118 , respectively ( $p>.15$ in all cases). These correlations do not support the idea that the experimental items with higher animalness ratings should have slower and less accurate responses than do the experimental items with lower animalness ratings.

In every respect, then, the results of Experiment 3 , which employed a within-subjects design, replicated the important findings of Experiments 1 and 2. First, the effect of semantic distance was not significant in the yes/no task condition, whereas it was significant in the go/no-go task condition. Second, response latencies to the experimental items were again longer in the go/no-go task condition than in the yes/no task condition.

\section{GENERAL DISCUSSION}

Buchanan et al. (2001) were the first to describe a systematic assessment of the effect of semantic distance in visual word recognition. They reported a facilitatory effect of semantic distance in lexical decision, in that responses to low semantic distance words were faster than responses to high semantic distance words. We thought it important to examine this effect of semantic distance in a different word recognition task (see Andrews, 1997), particularly one that requires accessing semantics before responses can be made. We therefore chose Forster and Shen's (1996) 
animal/nonanimal semantic categorization task, because this task ostensibly requires the retrieval of a word's meaning to correctly classify the animal name and nonanimal name stimuli. In Experiment 1, we used a yes/no task condition, in which the participants were required to press one key if the item was an animal name and to press a different key if the item was not an animal name. We did not find an effect of semantic distance under these task conditions.

Recall that we pointed out an important procedural difference between the lexical decision and the yes/no semantic categorization tasks. Specifically, the experimental items that require yes responses in lexical decision require no responses in yes/no semantic categorization (see also Carreiras et al., 1997). We proposed that a way to make responses to the experimental items more like yes responses in the semantic categorization task was to employ a different procedure. We therefore used a go/no-go task condition in Experiment 2 in which the participants responded only to the experimental items (i.e., the nonanimal items). We assumed that this procedural change would make responses to the experimental items more like yes responses, because they were the only overt responses the participants made. Our expectation was that there would be an increased likelihood of observing an effect of semantic distance under these conditions. This expectation was realized. The effect of semantic distance was much stronger in the go/no-go task condition $(41 \mathrm{msec})$ than in the yes/no task condition $(15 \mathrm{msec})$. In addition, response latencies to the experimental items were longer under go/no-go task conditions than under yes/no task conditions. These results were then replicated in Experiment 3, in which semantic distance and task condition were manipulated within subjects. Importantly, semantic distance effects were shown to be independent of both subjective frequency ratings (e.g., Conley et al., 2001) and animalness ratings (Carreiras et al., 1997).

Recently, feedforward and feedback activation accounts have been used to explain semantic (and phonological)effects in lexical decision, naming, and semantic categorization (e.g., Balota et al., 1991; Hino \& Lupker, 1996; Hino et al., 2002; Pecher, 2001; Pexman \& Lupker, 1999; Pexman et al., 2002; Pexman, Lupker, \& Jared, 2001; Stone, Vanhoy, \& Van Orden, 1997; Ziegler, Montant, \& Jacobs, 1997). There are several important assumptions in this explanatory framework. First, distinct sets of units dedicated to processing orthographic, phonological, or semantic information are reciprocally interconnected. Second, activation from one (or more) of these sets of units can influence processing in the other set(s) of units, and the nature of the activation determines whether facilitatory or inhibitory effects are observed. Third, lexical decisions are based on processing in the orthographic units, pronunciations are based on processing in the phonological units, and semantic categorizations are based on processing in the semantic units.

Buchanan et al. (2001) noted that low semantic distance words have denser semantic neighborhoods than do high semantic distance words, because they have, on average, more semantic neighbors within a specified radius. They suggested that this greater semantic processing for low semantic distance words would result in enhanced feedback activation from the semantic units to the orthographic units and, hence, faster lexical decision response latencies for these words. This was what they reported.

This feedback activation account is not tenable for our semantic categorization results, because semantic categorizations must be based on processing in the semantic units. How, then, does semantic distance influence processing in the semantic units? We suggest that semantic distance may have effects on semantic processing that are similar to those orthographic neighborhoods have on orthographic processing. Sears, Lupker, and Hino (1995; see also Andrews, 1992) suggested that connectionist models (e.g., Plaut, McClelland, Seidenberg, \& Patterson, 1996; Seidenberg \& McClelland, 1989) offer an elegant account of orthographic neighborhood effects. Since orthographically similar words would tend to recruit similar units and connections dedicated to orthographic processing, a word's orthographic representation would be strengthened not only when the word itself is presented, but also when its orthographic neighbors are presented. Thus, words with many orthographic neighbors would have their representations strengthened to a greater degree than would words with few orthographic neighbors. Sears, Hino, and Lupker (1999) conducted a series of statistical analyses of the orthographic, phonological, and cross-entropy error scores of the four- and five-letter monosyllabic words in the Plaut et al. and the Seidenberg and McClelland word corpi. They reported that words with many orthographic neighbors (and words with higher frequency orthographic neighbors) had, on average, lower error scores than did words with few orthographic neighbors (and words with no higher frequency neighbors).

We offer a similar account for the effect of semantic distance. Because semantically similar words would tend to recruit similar units and connections dedicated to semantic processing, a word's semantic representation would be strengthened when it and its semantic neighbors are presented (see also McRae et al., 1997). Therefore, because low semantic distance words have more semantic neighbors than do high semantic distance words, they would have stronger and richer semantic representations and would thus benefit more during processing.

One remaining issue is why the effect of semantic distance was more robust in the go/no-go task condition than in the yes/no task condition. If a discrete stage model of semantic categorization performance is assumed, both the yes/no and the go/no-go task conditions involve two distinct and temporally successive processing stages in responding (see Perea, Rosa, \& Gomez, 2002, for an analysis of processing stages in the yes/no and the go/no-go conditions in lexical decision). The first is a lexical processing stage, in which the meaning of a word is retrieved. The second is a response processing stage, in which a response is chosen.

Owing to the nature of this particular task (i.e., to classify exemplar items and nonexemplar items for a single category), there most likely existed a bias to make animal 
responses. Some support for this position is that in the yes/no task condition, responses to the animal name items were an average of $48 \mathrm{msec}$ faster, but $2 \%$ less accurate, than responses to the experimental items $\left[F_{1}(1,35)=5.52\right.$, $M S_{\mathrm{e}}=7,683.45 ; F_{2}(1,118)=27.61, M S_{\mathrm{e}}=2,592.39$, and $F_{1}(1,35)=4.73, M S_{\mathrm{e}}=16.09 ; F_{2}(1,118)=3.09, M S_{\mathrm{e}}=$ $35.01, p=.08$, respectively]. In addition, in the go/no-go task condition, responses to the animal name items were $5 \%$ less accurate than responses to the experimental items $\left[F_{1}(1,35)=17.07, M S_{\mathrm{e}}=27.43 ; F_{2}(1,118)=23.26, M S_{\mathrm{e}}=\right.$ 36.24]. Importantly, these findings were limited to task effects and do not compromise the effect of semantic distance that is central to this study. However, we believe that they are informative with respect to the question at hand.

Because of this animal response bias in the two task conditions, we propose that more extensive semantic processing of the experimental items was conducted during the lexical processing stage in the go/no-go task condition. The primary reason for this is because in the go/nogo task condition, the only overt responses required were to the experimental items. To ensure that correct responses were made, the participants may have set a higher lexical processing criterion before making a response and, thus, performed more extensive semantic processing of the experimental words to make sure they were indeed not animal names. This would have led to longer response latencies and lower error rates for the experimental items in the go/no-go task condition, as compared with the yes/no task condition. This is exactly what was observed in Experiment 3. Recall that in the analyses of the response latency and error rate data, the main effects of task procedure were significant. Response latencies were longer and error rates were lower to the experimental items in the go/no-go task condition than in the yes/no task condition.

But this was more so for the high semantic distance words than for the low semantic distance words. These data are consistent with the explanation offered above regarding how semantic distance influences semantic processing. Specifically, because the low semantic distance words have more semantic neighbors than do the high semantic distance words, they should have stronger and richer semantic representations (i.e., their connections have received greater strengthening, owing to the presentation of more words that are similar in meaning). Thus, the low semantic distance words are the very items that should benefit more from increased semantic processing. That is why these items have smaller increases in response latencies going from the yes/no task condition to the go/no-go task condition.

Finally, the two task conditions may have also differed in the processing demands required at the responseprocessing stage. In the yes/no task condition, participants must make two distinct responses and, moreover, must remember the correct stimulus-to-response pairing for the two stimuli classes. In the go/no-go task conditions, the response selection process is putatively simpler (Gibbs \& Van Orden, 1998; Perea et al., 2002). Under these conditions, participants need respond only to one class of stimuli and remember only one stimulus-to-response pairing. This analysis predicts that response latencies would be faster in the go/no-go task condition because of the reduced processing demands at the response selection stage.

Perea et al. (2002) reported faster response latencies (and lower error rates) in their go/no-go lexical decision condition than in their yes/no lexical decision condition. They noted that this replicates the findings of some researchers (e.g., Chiarello, Nuding, \& Pollock, 1988; Gordon \& Caramazza, 1982; Measso \& Zaidel, 1990; Perea, Fernandez, \& Carreiras, 1998) but not of others (e.g., Gibbs \& Van Orden, 1998; Hino \& Lupker, 1998, 2000). At this point, it may be premature to make firm conclusions about whether the reduced response selection demands of the go/no-go task condition necessarily leads to faster response latencies than do those of the yes/no task condition. Recall that the opposite was true for our study.

There is one important procedural difference between the lexical decision and the animal/nonanimal semantic categorization tasks that could account for the disparity between Perea et al.'s (2002) findings of faster response latencies and our results of slower response latencies in go/no-go conditions. The items responded to in Perea et al.'s (2002) lexical decision go/no-go condition were the exemplars of the task (i.e., words are exemplars of the category lexical items). This was not the case in our study. Even though we have suggested that requiring overt responses to the nonanimal items made these responses more like yes responses, they were still nonexemplars of the category animal. We wondered whether we would observe faster response latencies to the exemplar items (i.e., the animal name items) of our study if we conducted another experiment in which these items required the overt response in the go/no-go task condition. We used the same items and procedures as those in Experiment 3 to investigate this possibility. Sixteen participants received the yes/no task condition first and the go/no-go task condition second, whereas 16 participants received the go/no-go task condition first and the yes/no task condition second. The only difference was that the participants were instructed to respond only to the animal name items in the go/no-go task condition.

Responses to the animal name items were an average of $32 \mathrm{msec}$ faster and $2.3 \%$ more accurate in the go/no-go task condition than in the yes/no task condition $\left[F_{1}(1,31)=\right.$ $4.61, M S_{\mathrm{e}}=3,673.99, F_{2}(1,59)=12.79, M S_{\mathrm{e}}=2,233.13$, and $F_{1}(1,31)=11.47, M S_{\mathrm{e}}=7.31, F_{2}(1,59)=12.44, M S_{\mathrm{e}}$ $=12.82$, respectively]. These data, in combination with those of Experiment 3, indicate that responses are faster to the exemplar items of a task in the go/no-go task condition but are slower to the nonexemplar items. A prediction, based on these results, is that if nonwords were the items responded to in a go/no-go lexical decision task, response latencies would increase (as compared with response latencies to the same nonwords in a yes/no lexical decision task), because participants would process them more extensively in order to ensure that the correct responses were made.

In conclusion, the results of the present study, along with the results of Buchanan et al. (2001), provide converging evidence that semantic distance facilitates word recognition processes. In addition, we have suggested that 
as far as animal/nonanimal semantic categorization is concerned (and presumably, any other semantic categorization task that uses a specific and narrow classification of the stimuli), the go/no-go task condition allows for better comparisons between lexical decision and semantic categorization data than does the yes/no semantic categorization condition. We are currently investigating other variants of the semantic categorization task to obtain a better understanding of how these differences arise as a function of task condition. Finally, Balota et al. (1991), in their review of the effects of semantics in word recognition, did not report any semantic categorization tasks (or other tasks requiring semantic retrieval) that had been used to examine semantic effects in visual word recognition. The present study, along with other recent studies (e.g., Forster, 1999; Gottlob, Goldinger, Stone, \& Van Orden, 1999; Hino et al., 2002; Piercey \& Joordens, 2000), begins to fill this void in the literature regarding the use of word recognition tasks, such as semantic categorization, in which the retrieval of meaning is required in order to examine the effects of semantics in visual word recognition.

\section{REFERENCES}

Abrams, R. A. (1995). Psych/Lab experimental psychology software [Computer software]. St. Louis: Psych/Lab Software. (Available from Washington University Web site: ttp://www.artsci.wustl.edu/ rabrams/ psychlab/).

ANDREWs, S. (1992). Frequency and neighborhoodeffects on lexical access: Lexical similarity or orthographic redundancy? Journal of Experimental Psychology: Learning, Memory, \& Cognition, 18, 234-254.

ANDREWS, S. (1997). The effect of orthographic similarity on lexical retrieval: Resolving neighborhood conflicts. Psychonomic Bulletin \& Review, 4, 439-461.

Andrews, S., \& Heathcote, A. (2001). Distinguishing common and task-specific processes in word identification: A matter of some moment? Journal of Experimental Psychology: Learning, Memory, \& Cognition, 27, 514-544.

BaAyen, R. H., Piepenbrock, R., \& Gulikers, L. (1995). The CELEX Lexical Database [CD-ROM]. Philadelphia: University of Pennsylvania, Linguistic Data Consortium.

Balota, D. A., \& Chumbley, J. I. (1984). Are lexical decisions a good measure of lexical access? The role of word frequency in the neglected decision stage. Journal of Experimental Psychology: Human Perception \& Performance, 10, 340-357.

Balota, D. A., \& Chumbley, J. I. (1990). Where are the effects of frequency in visual word recognition tasks? Right where we said they were! Comment on Monsell, Doyle, and Haggard (1989). Journal of Experimental Psychology: General, 119, 231-237.

Balota, D. A. Ferraro, F. R., \& Connor, L. T. (1991). On the early influence of meaning in word recognition: A review of the literature. In P. J. Schwanenflugel(Ed.), The psychology of word meanings (pp. 187222). Hillsdale, NJ: Erlbaum.

Balota, D. A., Pilotti, M., \& Cortese, M. J. (2001). Subjective frequency estimates for 2,938 monosyllabic words. Memory \& Cognition, 29, 639-647.

BorowsKy, R. \& Masson, M. E. J. (1996). Semantic ambiguity effects in word identification. Journal of Experimental Psychology: Learning, Memory, \& Cognition, 22, 63-85.

Bradley, D. A., \& Forster, K. I. (1987). A reader's view of listening. Cognition, 25, 103-134.

Buchanan, L., Westbury, C., \& Burgess, C. (2001). Characterizing semantic space: Neighborhood effects in word recognition. Psychonomic Bulletin \& Review, 8, 531-544.

BuRGESS, C. (1998). From simple associations to the building blocks of language: Modeling meaning in memory with the HAL model. $B e-$ havior Research Methods, Instruments, \& Computers, 30, 188-198.
Burgess, C., Livesay, K., \& Lund, K. (1998). Explorations in context space: Words, sentences, discourse. Discourse Processes, 25, 211-257. BuRgESS, C., \& LunD, K. (2000). The dynamics of meaning in memory. In E. Dietrich \& A. B. Markman (Eds.), Cognitive dynamics: Conceptual and representational change in humans and machines (pp. 117156). Mahwah, NJ: Erlbaum.

Carreiras, M., Perea, M., \& Grainger, J. (1997). Effects of orthographic neighborhood in visual word recognition: Cross-task comparisons. Journal of Experimental Psychology: Learning, Memory, \& Cognition, 23, 857-871.

Chiarello, C., Nuding, S., \& Pollock, A. (1988). Lexical decision and naming asymmetries: Influence of response selection and response bias. Brain \& Language, 34, 302-314.

Coltheart, M. (1978). Lexical access in simple reading tasks. In G. Underwood (Ed.), Strategies of information processing (pp. 151-216). New York: Academic Press.

Coltheart, M. (1981). The MRC psycholinguistic database. Quarterly Journal of Experimental Psychology, 33A, 497-505.

Coltheart, M., Davelaar, E., Jonasson, J. T., \& Besner, D. (1977). Access to the internal lexicon. In S. Dornic (Ed.), Attention and performance VI (pp. 535-555). Hillsdale, NJ: Erlbaum.

Coltheart, M., Rastle, K., Perry, C., Langdon, R, \& Ziegler, J. (2001).DRC: A dual route cascaded model of visual word recognition and reading aloud. Psychological Review, 108, 204-256.

Conley, P., Burgess, C., \& Decker, C. (2001, November). An examination of semantic density and familiarity in semantic priming. Poster presented at the 42nd Annual Meeting of the Psychonomic Society, Orlando, FL.

Cortese, M. J., Simpson, G. B., \& Woolsey, S. (1997). Effects of association and imageability on phonological mapping. Psychonomic Bulletin \& Review, 4, 226-231.

Forster, K. I. (1999, November). Beyond lexical decision: Lexical access in categorization tasks. Paper presented at the 40th Annual Meeting of the Psychonomic Society, Los Angeles.

Forster, K. I., \& ShEN, D. (1996). No enemies in the neighborhood: Absence of inhibitory effects in lexical decision and semantic categorization. Journal of Experimental Psychology: Learning, Memory, \& Cognition, 22, 696-713.

GibBS, P., \& VAN Orden, G. C. (1998). Pathway selection's utility for control of word recognition. Journal of Experimental Psychology: Human Perception \& Performance, 24, 1162-1187.

Gordon, B., \& CARAmazzA, A. (1982). Lexical decision for open- and closed-class words: Failure to replicate differential frequency sensitivity. Brain \& Language, 15, 143-160.

Gottlob, L. R., Goldinger, S. D., Stone, G. O., \& Van Orden, G. C. (1999). Reading homographs: Orthographic, phonologic, and semantic dynamics. Journal of Experimental Psychology: Human Perception \& Performance, 25, 561-574.

Grainger, J., \& JACOBS, A. M. (1996). Orthographic processing in visual word recognition: A multiple read-out model. Psychological Review, 103, 518-565.

Green, P. E. (1978). Analyzing multivariate data. Hinsdale, IL: Dryden.

Hino, Y., \& LupKer, S. J. (1996). Effects of polysemy in lexical decision and naming: An alternative to lexical access accounts. Journal of Experimental Psychology: Human Perception \& Performance, 22, $1331-1356$

HiNO, Y., \& LUPKER, S. J. (1998). The effects of word frequency for Japanese Kana and Kanji words in naming and lexical decision: Can the dual-route model save the lexical selection account? Journal of Experimental Psychology: Human Perception \& Performance, 24, 1431-1453.

Hino, Y., \& LuPKER, S. J. (2000). The effects of word frequency and spelling-to-sound regularity in naming with and without lexical decision. Journal of Experimental Psychology: Human Perception \& Performance, 26, 166-183.

Hino, Y., Lupker, S. J., \& Pexman, P. M. (2002). Ambiguity and synonymy effects in lexical decision, naming, and semantic categorization tasks: Interactions between orthography, phonology, and semantics. Journal of Experimental Psychology: Learning, Memory, \& Cognition, 28, 686-713.

JAMES, C. T. (1975). The role of semantic information in lexical decisions. Journal of Experimental Psychology: Human Perception \& Performance, 1, 130-136. 
Kellas, G., Ferraro, F. R., \& Simpson, G. B. (1988). Lexical ambiguity and the timecourse of attentional allocation in word recognition. Journal of Experimental Psychology: Human Perception \& Performance, 14, 601-609.

Kroll, J. F., \& Mervis, J. S. (1986). Lexical access for concrete and abstract words. Journal of Experimental Psychology: Learning, Memory, \& Cognition, 12, 92-107.

Landauer, T. K., \& Dumais, S. T. (1997). A solution to Plato's problem: The latent semantic analysis theory of acquisition, induction, and representation of knowledge. Psychological Review, 104, 211-240.

Lund, K., \& Burgess, C. (1996). Producing high-dimensional semantic spaces from lexical co-occurrence. Behavior Research Methods, Instruments, \& Computers, 28, 203-208.

McRae, K., DE SA, V. R. \& Seidenberg, M. S. (1997). On the nature and scope of featural representations of word meaning. Journal of Experimental Psychology: General, 126, 99-130.

MeAsso, G., \& ZaIDEL, E. (1990). Effect of response programming on hemispheric differences in lexical decision. Neuropsychologia, $\mathbf{2 8}$, 635-646.

Monsell, S. (1990). Frequency effects in lexical tasks: Reply to Balota and Chumbley. Journal of Experimental Psychology: General, 119, 335-339.

Monsell, S., Doyle, M. C., \& Haggard, P. N. (1989). Effects of frequency on visual word recognition tasks: Where are they? Journal of Experimental Psychology: General, 118, 43-71.

Nelson, D. L., McEvoy, C. L., \& Schreiber, T. A. (1994). The University of South Florida word association, rhyme and word fragment norms. Unpublished manuscript.

Pecher, D. (2001). Perception is a two-way junction: Feedback semantics in word recognition. Psychonomic Bulletin \& Review, 8, 545-551.

Pedhazur, E. J. (1982). Multiple regression in behavioral research: Explanationand prediction (2nd ed.). New York: Holt, Rinehart \& Winston.

Perea, M., Fernandez, L., \& Carreiras, M. (1998, December). Efectos secuenciales en la tarea de decisión léxica [Sequential effects in the lexical decision task]. Paper presented at the II Conference of the Spanish Experimental Psychology Society, Granada.

Perea, M., Rosa, E., \& Gomez, C. (2002). Is the go/no-go lexical decision task an alternative to the yes/no lexical decision task? Memory \& Cognition, 30, 34-45.

Pexman, P. M., \& LuPKer, S. J. (1999). Ambiguity and visual word recognition: Can feedback explain both homophone and polysemy effects? Canadian Journal of Experimental Psychology, 53, 323-334.

Pexman, P. M., Lupker, S. J., \& Hino, Y. (2002). The impact of feedback semantics in visual word recognition: Number-of-features effects in lexical decision and naming tasks. Psychonomic Bulletin \& Review, 9, 542-549.
Pexman, P. M., Lupker, S. J., \& Jared, D. (2001). Homophone effects in lexical decision. Journal of Experimental Psychology: Learning, Memory, \& Cognition, 27, 139-156.

Piercey, C. D., \& Joordens, S. (2000). Turning an advantage into a disadvantage: Ambiguity effects in lexical decision versus reading tasks. Memory \& Cognition, 28, 657-666.

Plaut, D. C., McClelland, J. L., Seidenberg, M. S., \& Patterson, K. (1996). Understanding normal and impaired reading: Computational principles in quasi-regular domains. Psychological Review, 103, 56-115.

SCHNEIDER, W. (1990). Micro Experimental Laboratory [Computer software]. Pittsburgh: Psychology Software Tools.

SeArs, C. R., Hino, Y., \& LuPKeR, S. J. (1999). Orthographic neighbourhood effects in parallel distributed processing models. Canadian Journal of Experimental Psychology, 53, 220-229.

Sears, C. R., Lupker, S. J., \& Hino, Y. (1995). Neighborhood size and neighborhood frequency effects in word recognition. Journal of Experimental Psychology: Human Perception \& Performance, 21, 876-900.

SeArs, C. R, LupKer, S. J., \& Hino, Y. (1999). Orthographic neighborhood effects in perceptual identification and semantic categorization tasks: A test of the multiple read-out model. Perception \& Psychophysics, 61, 1537-1554.

Seidenberg, M. S., \& McClelland, J. L. (1989). A distributed, developmental model of word recognition and naming. Psychological Review, 96, 521-568.

Stone, G. O., Vanhoy, M., \& Van Orden, G. C. (1997). Perception is a two-way street: Feedforward and feedback phonology in visual word recognition. Journal of Memory \& Language, 36, 337-359.

Strain, E., Patterson, K., \& Seidenberg, M. S. (1995). Semantic effects in single word naming. Journal of Experimental Psychology: Learning, Memory, \& Cognition, 21, 1140-1154.

Toglia, M. P., \& Battig, W. F. (1978). Handbook of semantic word norms. Hillsdale, NJ: Erlbaum.

Ziegler, J. C., Montant, M., \& Jacobs, A. M. (1997). The feedback consistency effect in lexical decision and naming. Journal of Memory \& Language, 37, 533-554

\section{NOTES}

1. We thank Michael Cortese for making these data available to us.

2. The concreteness and imageability ratings from the MRC psycholinguistic database were multiplied by 100 to give scores from 100 to 700 , since the original ratings were taken from questionnaires that had values from 1 to 7 . In line with this data transformation, the concreteness and imageability ratings for the 38 words taken from Experiment 1 were also multiplied by 100 . Thus, the different values for these two variables in Table 5, as compared with Table 3. 
APPENDIX A

Mean Item Response Latencies (in Milliseconds) in Experiments 1 and 2

\begin{tabular}{|c|c|c|c|c|c|}
\hline \multicolumn{3}{|c|}{ High Semantic Distance Items } & \multicolumn{3}{|c|}{ Low Semantic Distance Items } \\
\hline Word & Experiment 1 & Experiment 2 & Word & Experiment 1 & Experiment 2 \\
\hline$\overline{\text { bulb }}$ & 694 & 681 & bike & 638 & 607 \\
\hline dusk & 658 & 723 & blade & 637 & 633 \\
\hline frail & 741 & 707 & blame & 618 & 688 \\
\hline fuss & 682 & 664 & brake & 658 & 643 \\
\hline globe & 602 & 632 & bunch & 668 & 688 \\
\hline halt & 644 & 701 & chess & 669 & 659 \\
\hline junk & 612 & 623 & cliff & 645 & 644 \\
\hline ledge & 641 & 658 & drum & 604 & 651 \\
\hline noon & 667 & 689 & fake & 651 & 683 \\
\hline oath & 731 & 814 & foam & 672 & 762 \\
\hline porch & 698 & 694 & hang & 603 & 643 \\
\hline purse & 639 & 646 & hurt & 595 & 617 \\
\hline rung & 634 & 715 & plug & 660 & 612 \\
\hline scalp & 686 & 675 & shake & 818 & 836 \\
\hline shrub & 705 & 718 & $\operatorname{sing}$ & 675 & 641 \\
\hline slum & 689 & 733 & spin & 713 & 676 \\
\hline spine & 774 & 835 & spray & 746 & 661 \\
\hline stake & 733 & 800 & stuck & 665 & 636 \\
\hline swift & 659 & 674 & teach & 595 & 623 \\
\hline tact & 635 & 702 & twin & 711 & 671 \\
\hline
\end{tabular}

\section{APPENDIX B}

\section{Animal Names Used in Experiments 1 and 2}

Four-letter animal names: bear, bees, bull, clam, cows, crow, deer, dove, duck, frog, goat, lamb, lion, lynx, mule, pigs, pony, swan, tuna, wolf.

Five-letter animal names: camel, chimp, eagle, goose, horse, mouse, otter, puppy, raven, roach, robin, shark, sheep, skunk, snail, snake, stork, tiger, whale, zebra.

\section{Animal Names Used in Experiment 3}

Four-letter animal names: ants, bats, bear, bees, bull, calf, clam, colt, cows, crow, deer, dove, duck, fawn, flea, fowl, frog, goat, gull, hawk, lamb, lion, loon, lynx, mole, mule, newt, owls, pigs, pony, puma, swan, tuna, wasp, wolf, worm.

Five-letter animal names: camel, chimp, eagle, goose, horse, hyena, koala, mouse, otter, panda, puppy, raven, roach, robin, $\underline{\text { shark, sheep, skunk, snail, snake, stork, tiger, trout, whale, zebra. }}$ 
APPENDIX C

Mean Item Response Latencies (in Milliseconds) in the Yes/No and Go/No-Go Task Procedures in Experiment 3

\begin{tabular}{|c|c|c|c|c|c|}
\hline \multicolumn{3}{|c|}{ High Semantic Distance Items } & \multicolumn{3}{|c|}{ Low Semantic Distance Items } \\
\hline Word & Yes/No & $\overline{\mathrm{Go} / \mathrm{No}-\mathrm{Go}}$ & Word & $\mathrm{Yes} / \mathrm{No}$ & $\mathrm{Go} / \mathrm{No}-\mathrm{Go}$ \\
\hline$\overline{\text { bulb }}$ & 742 & 687 & bike & 690 & 699 \\
\hline cape & 693 & 802 & blade & 694 & 609 \\
\hline crumb & 655 & 618 & blame & 612 & 631 \\
\hline dime & 675 & 662 & brake & 673 & 630 \\
\hline dusk & 661 & 796 & bunch & 736 & 610 \\
\hline frail & 640 & 818 & burn & 620 & 711 \\
\hline fuss & 644 & 673 & chess & 704 & 763 \\
\hline globe & 641 & 729 & cliff & 639 & 661 \\
\hline halt & 647 & 671 & craft & 771 & 688 \\
\hline itch & 694 & 747 & drum & 658 & 640 \\
\hline junk & 633 & 741 & fake & 638 & 674 \\
\hline ledge & 688 & 719 & foam & 690 & 694 \\
\hline limb & 744 & 859 & graph & 719 & 717 \\
\hline meek & 765 & 918 & hang & 608 & 676 \\
\hline noon & 719 & 726 & hide & 668 & 737 \\
\hline oath & 785 & 787 & hurt & 614 & 695 \\
\hline porch & 731 & 743 & kite & 739 & 727 \\
\hline purse & 666 & 650 & loop & 645 & 730 \\
\hline rung & 730 & 804 & pick & 745 & 678 \\
\hline scalp & 684 & 753 & plot & 609 & 632 \\
\hline shrub & 782 & 817 & plug & 670 & 725 \\
\hline sink & 722 & 774 & punch & 797 & 706 \\
\hline slum & 713 & 809 & sail & 803 & 868 \\
\hline sock & 667 & 669 & sing & 631 & 689 \\
\hline spine & 745 & 862 & spin & 641 & 699 \\
\hline spoon & 677 & 644 & spray & 624 & 706 \\
\hline swift & 694 & 669 & stuck & 647 & 751 \\
\hline tact & 662 & 703 & teach & 612 & 631 \\
\hline tense & 584 & 695 & tire & 612 & 650 \\
\hline toil & 674 & 692 & twin & 720 & 760 \\
\hline
\end{tabular}

(Manuscript received October 29, 2001;

revision accepted for publication September 26, 2002.) 